\title{
Patient involvement in preparing health research peer-reviewed publications or results summaries: a systematic review and evidence-based recommendations
}

\author{
Lauri Arnstein ${ }^{1 *}$, Anne Clare Wadsworth ${ }^{1,2}$, Beverley Anne Yamamoto ${ }^{3,4,5}$, Richard Stephens ${ }^{6,7}$, Kawaldip Sehmi $^{8}$, \\ Rachel Jones ${ }^{9}$, Arabella Sargent ${ }^{10}$, Thomas Gegeny ${ }^{11}$ and Karen L. Woolley ${ }^{1,12,13,14}$ (D)
}

\begin{abstract}
Background: There are increasing calls for patient involvement in sharing health research results, but no evidencebased recommendations to guide such involvement. Our objectives were to: (1) conduct a systematic review of the evidence on patient involvement in results sharing, (2) propose evidence-based recommendations to help maximize benefits and minimize risks of such involvement and (3) conduct this project with patient authors.

Methods: To avoid research waste, we verified that no systematic reviews were registered or published on this topic. We co-created, with patients, a PRISMA-P-compliant protocol. We included peer-reviewed publications reporting the effects of patient involvement in preparing peer-reviewed publications or results summaries from health research studies. We searched (9/10/2017) MEDLINE, EMBASE and the Cochrane Database of Systematic Reviews, and secondary information sources (until 11/06/2018). We assessed the risk of bias in eligible publications and extracted data using standardized processes. To evaluate patient involvement in this project, we co-created a Patient Authorship Experience Tool.

Results: All nine eligible publications reported on patient involvement in preparing publications; none on preparing results summaries. Evidence quality was moderate. A qualitative synthesis of evidence indicated the benefits of patient involvement may outweigh the risks. We have proposed 21 evidence-based recommendations to help maximize the benefits and minimize the risks when involving patients as authors of peer-reviewed publications. The recommendations focus on practical actions patient and non-patient authors can take before (10 recommendations), during (7 recommendations) and after (4 recommendations) manuscript development. Using the Patient Authorship Experience Tool, both patient and non-patient authors rated their experience highly.
\end{abstract}

Conclusions: Based on a systematic review, we have proposed 21 evidence-based recommendations to help maximize the benefits and minimize the risks of involving patients as authors of peer-reviewed publications.

Keywords: Patient and public involvement, Patient participation, PPI, Research reporting, Systematic review, Authorship, Medical writing, Clinical trials, Health research, Patient author

\footnotetext{
* Correspondence: lauri.arnstein@envisionpharmagroup.com

${ }^{1}$ Envision the Patient - Envision Pharma Group, Suite 5.11, 5th Floor, 1 Lyric

Square, London W6 ONB, UK

Full list of author information is available at the end of the article
}

\section{$\triangle B M C$}

(c) The Author(s). 2020 Open Access This article is licensed under a Creative Commons Attribution 4.0 International License, which permits use, sharing, adaptation, distribution and reproduction in any medium or format, as long as you give appropriate credit to the original author(s) and the source, provide a link to the Creative Commons licence, and indicate if changes were made. The images or other third party material in this article are included in the article's Creative Commons licence, unless indicated otherwise in a credit line to the material. If material is not included in the article's Creative Commons licence and your intended use is not permitted by statutory regulation or exceeds the permitted use, you will need to obtain permission directly from the copyright holder. To view a copy of this licence, visit http://creativecommons.org/licenses/by/4.0/ The Creative Commons Public Domain Dedication waiver (http://creativecommons.org/publicdomain/zero/1.0/) applies to the data made available in this article, unless otherwise stated in a credit line to the data. 


\section{Plain language summary}

- This research project is a systematic literature review. This is a structured way to look at all the published research on a topic.

- This review looked at patient involvement in sharing results from health research studies, including clinical trials. Results from health research studies are shared in different ways, such as:

- Being published in a scientific journal (called a peer-reviewed publication);

- Being presented in a lay summary (called a clinical trial results summary).

- The researchers wanted to know:

- What are the benefits and risks of involving patients in sharing results?

- What recommendations could help to increase the possible benefits and reduce the possible risks?

- What was the experience of patient authors taking part in this review project?

- The researchers searched for published research from different sources. They found 167 articles, of which nine were relevant.

- The researchers found that:

- All nine articles were about patient involvement in peer-reviewed publications. None of the articles were about patient involvement in clinical trial results summaries.

- The benefits of patient involvement in sharing results appeared to outweigh the risks.

- Based on the evidence they found, the researchers made 21 recommendations.

- These could help increase the possible benefits and reduce the possible risks when involving patients as authors of peer-reviewed publications.

- Overall, the patient and non-patient authors involved in this review project had a good experience.

- These results suggest that patients should be involved in sharing research results. There are now recommendations on how to involve patients as authors on publications.

See Additional file 1 for an infographic plain language summary.

\section{Background}

The importance of ethical and substantial patient involvement in medicines development is being acknowledged by stakeholders [1, 2]. Patients are becoming informed, empowered and active partners in their healthcare, and are calling for increased involvement across the entire medicines development life cycle [3, 4]. In response, stakeholders are recognizing the need for internal process and cultural change. Regulators, including the European Medicines Agency and the US Food and Drug Administration, have declared patient involvement to be a priority, and payers are increasingly taking the patient perspective into account in reimbursement decisions $[1,5]$. The pharmaceutical industry is beginning to empower the patient voice in medicines development, partnering with international patient advocacy organizations and healthcare professionals to establish ethical frameworks and principles to facilitate patient involvement [6-8].

In conjunction with this patient-empowered evolution, patients are increasingly seeking health information from peer-reviewed publications, and this demand is likely to increase $[9,10]$. Consistent with this interest from patients and their advocates, some medical journals are facilitating greater involvement of patients as authors, editors, peer reviewers and readers [11, 12]. In addition, increasing attention is being focused on involving patients in plain language summaries of publications of clinical trials and other types of research publications, building upon the European Medicine Agency's regulatory requirement for lay summaries of clinical trial results [13].

However, in comparison to patient involvement in other phases of the health research life cycle, such as study design and recruitment, patient involvement in publications has lagged behind. Not only does the level of patient involvement in publications seem low, but the potential benefits and risks of patient involvement are not well understood. Such insights are needed to define evidence-based best practices, thereby enhancing the potential benefits and minimizing the potential risks of involving patients as publication partners (e.g. as authors or contributors).

Our objectives were to: (1) conduct a systematic review of the evidence on patient involvement in results sharing, (2) propose evidence-based recommendations to help maximize benefits and minimize risks of such involvement and (3) conduct this project with patient authors.

\section{Methods}

\section{Protocol, registration and definition}

This systematic review was registered in the PROSPERO database (PROSPERO 2018 CRD42018084452), conducted according to a prespecified protocol and reported in compliance with best-practice reporting guidelines for systematic reviews [14] and research involving patients [15]. For this study, we employed the PICO model to define the key elements in our research, which included peer-reviewed publications that have investigated the effects of having patients ( $\mathrm{P}$ - Participants) engaged as authors of or contributors to (I - Intervention) peerreviewed publications. We quantified the number of 
these publications (Outcomes) and compared this with the number of publications on patient involvement in the preparation of lay summaries of clinical trial results (Comparator).

To minimize the risk of research waste, we searched (5 June 2017) the PROSPERO database to ensure we were not duplicating a planned or ongoing systematic review. We also registered our review on SYNAPSE, the Patient Focused Medicines Development (PFMD) repository for patient engagement initiatives (https://synapse.pfmd.org/initiatives/patient-involvement-in-preparing-clinical-researchpeer-reviewed-publications-or-results-summaries-a-systematic-review).

Within this study, 'patient' was defined in broad terms, based on an existing definition [16, 17] and input from our patient partners. For this research, 'patient' refers to "people having or at risk of having medical condition(s), whether or not they currently receive medicines or vaccines to prevent or treat a disease" as well as "the family and those voluntarily caring for those with the medical condition(s), patient advocates and patient groups."

\section{Eligibility criteria}

We included peer-reviewed publications that studied the effects of patient involvement for sharing results of health research studies (all phases and all designs) either through a peer-reviewed publication or through a lay summary of clinical trial results (i.e. consistent with the regulatory requirements described in the EU Clinical Trials Regulation 536/2014 [Article 37] [13]). We considered publications, in any language, that were detected in searches of electronic bibliographic databases or secondary sources (see Information sources and search strategies section). We did not include unpublished data or abstract-only articles.

\section{Information sources and search strategies}

We used primary and secondary information sources. For our primary source, we searched three electronic bibliographic databases (MEDLINE, EMBASE and Cochrane Database of Systematic Reviews) using the OVID database search interface. We used Medical Subject Headings (MeSH) and words/phrases related to patient involvement in health research peer-reviewed publications and lay summaries of clinical trial results. An experienced search strategist conducted the search of the three databases on 9 October 2017 (limits: 1 January 2015-9 October 2017; see Additional File 2 for full search strategies). References were exported to a reference management software program (EndNote) and saved into a project-specific library within EndNote. Duplicate publications were removed.

We supplemented our primary information source with secondary information sources. These sources comprised the repository of patient involvement in research literature curated by the Patient-Centered Outcomes Research Institute (PCORI), PubMed's 'similar articles' listings and the authors' personal reference files. A search of the PCORI repository was conducted on 10 June 2018 via PCORI's Engagement in Health Research Literature Explorer (limits: dissemination, 1 January 2017-10 June 2018); a search of the top 10 PubMed articles most related to eligible articles retrieved from our primary information sources was conducted on 11 June 2018; and authors' personal references were scanned up until 30 May 2018.

\section{Study selection}

We assessed publications for eligibility using a standardized process and a dedicated assessment team (three authors with predefined roles). One author (KW) selected potentially eligible articles based on a title and abstract screen, then two authors (LA, TG) independently selected eligible articles based on a review of the full text of each potential article. Differences in assessment were resolved by consensus among the assessment team.

\section{Data collection process and data items}

Based on the protocol, we prepared a spreadsheet template to collect data from all eligible publications. The template was pilot tested and refined before use. Data were collected using a standardized process and a dedicated data collection team (three authors with predefined roles). Two authors (RJ, AS) independently extracted data from each publication and a third author $(\mathrm{ACW})$ verified $100 \%$ of the data extracted for the primary outcome measure from every eligible publication, and verified $100 \%$ of the data extracted for the secondary outcome measures from a random sample of $25 \%$ of the eligible publications. Data (Additional File 3) were extracted for publication title, journal, year, authors, first author's country, research sponsor, number of patient authors, patient author background, patient author recruitment process, author communication process, extent of patient involvement in preparing the publication or lay summary of clinical trial results, how patient involvement outcomes were assessed, benefits (to patient authors, other authors, the project), risks (to patient authors, other authors, the project) and best practice recommendations.

\section{Risk of bias in individual studies}

We anticipated that there would be few randomized controlled trials that directly addressed our research question. As per the protocol, the Cochrane Collaboration tool for assessing bias risk (https://handbook-5-1. cochrane.org/chapter_8/table_8_5_a_the_cochrane_collaborations_tool_for_assessing.htm) was to be used to 
assess randomized controlled trials. The NewcastleOttawa Scale was used to evaluate the risk of bias for other study designs, although we recognize this instrument does have limitations (http://www.ohri.ca/programs/clinical_epidemiology/oxford.asp).

\section{Summary measures}

Our primary outcome measure was the number of peerreviewed publications that investigated the effect of patient involvement on preparing peer-reviewed publications.

Our secondary outcome measures were:

a. The number of peer-reviewed publications that investigated the effect of patient involvement on preparing regulatory-standard lay summaries of clinical trial results;

b. The quality of the evidence reported in the eligible publications;

c. The number and the background (e.g. patient experts, clinical trial participants, patient advocacy group members) of patients contributing to the preparation of the publications or lay summaries of clinical trial results;

d. The type of patient involvement (e.g. as authors, as non-author contributors);

e. The number and type of patient involvement outcomes assessed (e.g. benefits, risks, best practice recommendations, other).

\section{Synthesis of results}

As anticipated in the protocol, we did not synthesize data quantitatively due to heterogeneity in the eligible publications. We conducted a qualitative narrative synthesis of the data to describe patient involvement outcomes.

\section{Additional analyses}

To assess the quality of the patient authorship experience in the present study, one author (KW) customized the original PFMD Patient Engagement Quality Guidance [16], which PFMD co-created with over 100 people from 51 organizations, representing patient associations, industry, academics, researchers, and external experts. The resulting draft Patient Authorship Experience (PAE) self-assessment tool was then updated with co-author feedback. The final PAE tool evaluates eight publicationrelevant domains using a bipolar, five-point, psychometric, Likert scale (strongly disagree to strongly agree) [18]. The total score is determined by adding up the individual scores for each question and calculating a percentage out of a maximum score of 80 . Two versions were created: one for patient authors and another for nonpatient authors. All authors in this study completed the PAE tool.
To document the involvement of patients in this project, the GRIPP2 Short Form was completed [15].

\section{Results \\ Quantity of evidence on patient involvement}

The number of peer-reviewed publications that reported the effects of patient involvement on preparing peerreviewed publications was low. An extensive search of primary and secondary information sources yielded only nine publications (Fig. 1). Of these nine publications, nine reported on the effects of patient involvement in preparing peer-reviewed publications; none reported on the effects of patient involvement in preparing regulatory-standard lay summaries of clinical trial results.

\section{Quality of evidence on patient involvement}

The quality of evidence on patient involvement in preparing peer-reviewed publications was moderate. There were no published systematic reviews or randomized controlled trials focused on this topic. Assessments based on the Newcastle-Ottawa scale, indicated six of the nine studies were good quality and three were fair/ poor quality (Table 1).

\section{Type of patients involved and type of involvement}

Patients were involved as authors in six of nine publications, and as contributors in eight of nine publications; the majority of them were members of patient and public involvement (PPI) groups (Table 1).

\section{Evidence on benefits and risks of patient involvement}

From the evidence reported, patient involvement was associated with benefits and risks, which we categorized into those that affected: (1) patients, (2) others (primarily researchers and clinicians) and (3) the actual research projects (Table 2). Based on the number of effects and the overall impact, the potential benefits of patient involvement appear to outweigh the potential risks. Key benefits include validation of research need, relevance and value; improved materials and processes; improved reporting and dissemination; enhanced credibility and trust; and development of new knowledge and skills. Risks focused on increased resource needs and opportunity costs; navigation of power dynamics; and risk of nonrepresentative insights from less diverse PPI groups.

\section{Evidence on best practices recommendations for patient involvement}

Based on the evidence extracted from the eligible publications (Additional file 3), we developed a list of $21 \mathrm{rec}-$ ommendations that could help maximize the benefits and minimize the risks of involving patients in preparing peer-reviewed publications (Table 3). Each recommendation is linked to the eligible publications that provided 


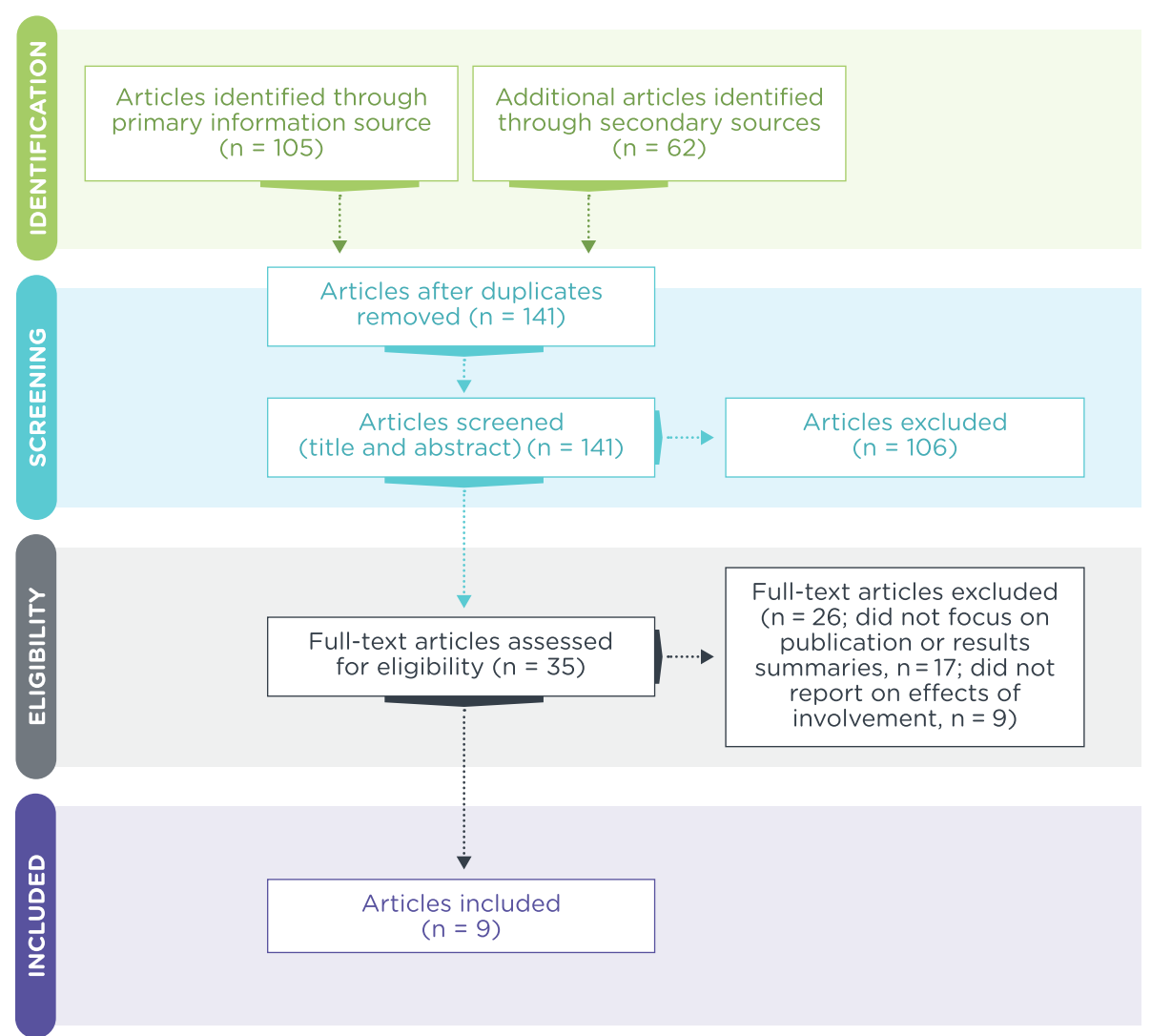

Fig. 1 Flow of screened and eligible publications (PRISMA Flow Diagram)

the evidence to support that particular recommendation. To enhance practical use, the recommendations are categorized into actions that research teams could take before, during and after manuscript preparation.

\section{PAE self-assessment tool}

Overall, authors in this study rated their co-authorship experience highly. The average PAE tool score was $86 \%$ for patient authors (69 of $80 ; \mathrm{N}=3$ ) and $90 \%$ for nonpatient authors ( 72 of $80 ; \mathrm{N}=6$ ). The authors identified areas to enhance future collaboration; for example, fewer assumptions on the part of the research team about patient authors' prior experience and knowledge of publications and greater awareness of the extent of patients' emotional investment in research involvement.

\section{Reporting of patient involvement in this project}

Consistent with best-practice reporting for patient involvement in research [15], the involvement of patient co-authors in this project has been documented using the GRIPP2 Short Form (Table 4).

\section{Discussion}

To our knowledge, this is the first systematic review of evidence on the effects of patient involvement in sharing results from health research studies. We identified nine publications that reported benefits and risks when patients were involved in sharing results through peer-reviewed publications. From this systematic review, we have proposed 21 evidence-based recommendations to help maximize the benefits and minimize the risks of involving patients as authors of peer-reviewed publications.

Consistent with the drive for greater involvement of patients in the development, regulation and reimbursement of medicines [1-4], there is growing recognition that patients should be more involved in sharing research results. However, in contrast to the proliferation of general guidelines on how to involve patients in the medicines life cycle $[2,3,6,7]$, there is limited guidance on how to involve patients in sharing results. Traditionally, results from all types of health research have been shared through peer-reviewed publications in medical journals. These publications have usually been authored by medical research scientists and clinicians. More recently, European regulatory requirements have prompted the development of lay summaries of results from certain types of clinical trial studies [13]. These regulatory-standard lay summaries are usually authored by research sponsors (e.g. pharmaceutical company staff or service providers). We reasoned that if patients are 


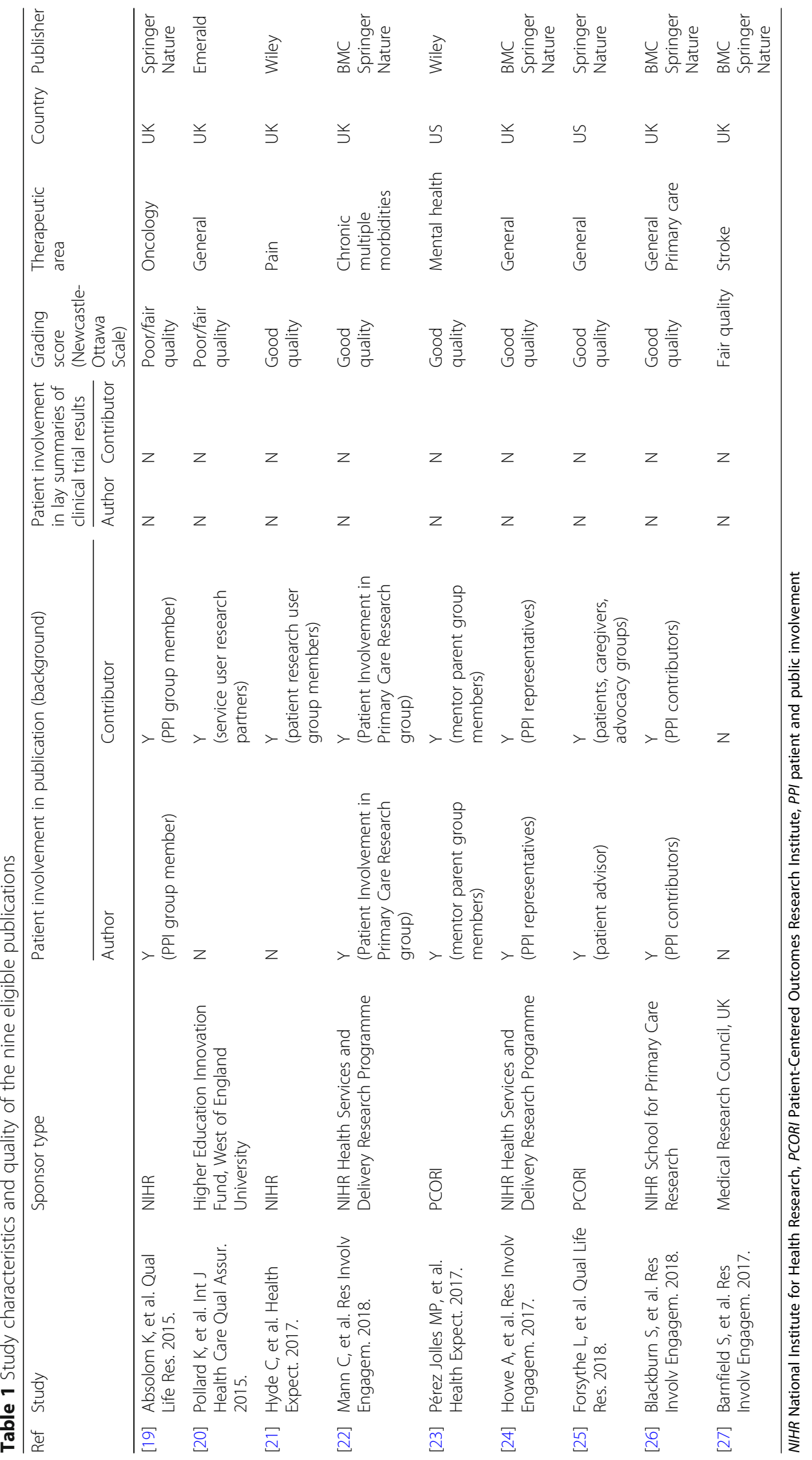


Table 2 Reported benefits and risks of involving patients in preparing peer-reviewed publications

\begin{tabular}{|c|c|c|c|c|c|c|}
\hline \multirow{2}{*}{$\begin{array}{l}\text { Reference } \\
\#\end{array}$} & \multicolumn{3}{|c|}{ Benefits of patient involvement } & \multicolumn{3}{|c|}{ Risks of patient involvement } \\
\hline & For patients & For others & For the project & For patients & For others & For the project \\
\hline $\begin{array}{l}{[19]} \\
\text { Absolom } \\
\text { et al. }\end{array}$ & $\begin{array}{l}\text { Feel that } \\
\text { contribution is } \\
\text { valued and actioned; } \\
\text { gain new skills and } \\
\text { knowledge of the } \\
\text { research process; } \\
\text { opportunity to use } \\
\text { transferrable skills; } \\
\text { increased confidence }\end{array}$ & $\begin{array}{l}\text { Enrich researcher } \\
\text { understanding of the } \\
\text { topic; learn how to } \\
\text { tailor projects } \\
\text { around patients' } \\
\text { availability, health } \\
\text { status and interest }\end{array}$ & $\begin{array}{l}\text { Patients raised new } \\
\text { issues, such as } \\
\text { sensitive data } \\
\text { management and } \\
\text { cultural diversity; } \\
\text { patients asked } \\
\text { questions that } \\
\text { produced useful } \\
\text { discussion }\end{array}$ & $\begin{array}{l}\text { May initially feel } \\
\text { daunted by } \\
\text { experienced } \\
\text { researchers and } \\
\text { clinicians; time } \\
\text { needed to get to } \\
\text { grips with complex } \\
\text { information }\end{array}$ & $\begin{array}{l}\text { Time investment to } \\
\text { brief patients before } \\
\text { meetings }\end{array}$ & $\begin{array}{l}\text { Patient withdrawal } \\
\text { due to ill health can } \\
\text { produce team gaps } \\
\text { and make it } \\
\text { challenging to } \\
\text { sustain a volunteer- } \\
\text { run committee in } \\
\text { the longer term }\end{array}$ \\
\hline
\end{tabular}

representative

[20] Improved confidence Gain insight

Pollard and skills, including early planning and

et al. facilitation,

handwriting and

speaking; feel

supported by having

an academic

researcher dedicated

to evaluating the

patient experience

[21] Opportunity to input Support in

Hyde

et al.

results reporting and

dissemination plan;

ensure that patient

priorities are directly

reflected in the

research; patients

plan their own roles

including content

review, attending

and giving

presentations and

contributing their

perspective at

conferences;

involvement is

valued and non-

tokenistic

[22] Enjoy being part of a

Mann group; gain

et al. confidence and

freedom to

challenge

researchers; learn

listening skills and

how to share views;

training

opportunities; sense

of ownership

towards the trial; feel

listened to and

valued; sense of

making a difference

patients identified consistent guideline

use around

enhancing patient

involvement are

important; gain

greater awareness of

challenges such as

meetings and events

timing, accessibility

and communication

Stakeholder diversity

in meetings

(academics,

practitioners and

patients)

patients may not feel

confident, or may

have difficulty

capturing the

group's attention;

logistical challenges,

such organizing

transport to and

from meetings

identifying new

research topics;

reassurance that

similar ideas (validity

of input) and new

ideas (innovative

Help ensure that the project is funded; refinement of the project scope by identifying gaps in the published

literature; results

interpretation and

input) to researchers; the dissemination

develop strategies to plan more clearly

help manage patient reflect patient

involvement

challenges and

establish best

practice

priorities

Navigation of time pressures and power dynamics; no

evaluation of the

experience of the

patients themselves

Navigation of time pressures and power dynamics

Other stakeholders may be cautious of patient involvement

Need to structure projects to address patients' needs (e.g. fatigue, mobility and communication concerns)

Best practice recommendations not validated by assessment of patient experiences; patient insights may not be fully representative, as the patient group was non-diverse and carers and stakeholder organizations were not involved; potential for introducing research bias
Gain insights from beyond the

academic world

with patients as a

'sounding board' and

'reality check';

improve training

course for trial

clinicians following

patient input; increase knowledge

of patient groups

and organizations;

learn communication

skills; sense of the

research being

worthwhile and

rewarding; more

favourable

perception of the

potential of patient
Improve documents and webpage used in the trial, including better accessibility;

gain advice on data collection and analysis methods; input into dissemination plan and initiation of publication; creation of an environment for substantial discussion and challenge

(n)

Conflict over differing participation requirements between patients: feel 'taken for granted' and perspectives not valued; communication challenges posed by patient group diversity; difficulty attending meetings due to illness, treatment, employment or other commitments; frustration at limited input into study design; nonconcordance
Defensiveness and lack of respect for patient perspectives; frustration at lack of patients' understanding of the constraints of the research, and at not being able to incorporate all of their good ideas; non-concordance between patient and researcher priorities
Patient insights may not be fully representative, as group had high literacy levels compared with the general population 
Table 2 Reported benefits and risks of involving patients in preparing peer-reviewed publications (Continued)

\begin{tabular}{ll}
\hline Reference & Benefits of patient inv \\
\cline { 2 - 2 } & For patients \\
\hline & \\
[23] & Sense of motivation \\
Pérez & and satisfaction at \\
Jolles & helping other \\
et al. & $\begin{array}{l}\text { patients and carers; } \\
\text { meetings planned to } \\
\text { fit around patients' } \\
\text { schedules; meetings } \\
\text { conducted in } \\
\text { preferred language; } \\
\text { remuneration for } \\
\text { participation }\end{array}$
\end{tabular}

[24] Opportunity for Howe training around et al. research and providing insights; growing confidence in role over time; gain new, rewarding skills; remuneration for participation; feel supported and valued

$\begin{array}{ll}\text { [25] } & \text { Satisfaction from } \\ \text { Forsythe } & \text { presenting and } \\ \text { et al. } & \text { generating interest } \\ & \text { in the research }\end{array}$

Growing confidence in role over time; help ensure accountability to funders and the public; successful relationship-building

For others
involvement; success
in overcoming
tokenism

Gain insights on language to use to build trust with patients

For the project

Recommendations to improve study recruitment and retention; validation of and improvements to primary outcome measure and study tool; validation of analytical approach; credible, patient-led presentation of results to broader community; COcreation of visuals to enhance research presentations and publication; recommendations for further research

Shaping of the

mproved skills to communicate with patients recruitment and Risks of patient involvement

\begin{tabular}{l}
\hline For patients $\quad$ For others $\quad$ For the project \\
$\begin{array}{l}\text { between patient and } \\
\text { researcher priorities }\end{array}$
\end{tabular}

Challenging for patients to fully participate in results dissemination, e.g. unable to travel to a national conference to present due to competing responsibilities
None reported

group came from
research process, including study documents and analysis methods; collection of new data; improved communication through anticipating accessibility

challenges; input into dissemination plan; development of a tool for reflective practice different roles (co-

Enhanced study retention; validation and innovation of research topics, interventions, outcomes and measures; adapt materials and interventions to be more culturally or linguistically appropriate; modify intervention to be less burdensome for patients; contribution to data collection; new ways to share results; new audiences to reach and improved communication with different audiences; increasing credibility
Tensions between patient members on the relative value of researcher vs. advisory committee); feeling 'on the periphery' in certain roles; feel 'lost' through jargon procedures and lack of support; accessibility challenges (literacy, mobility, distance, digital); remuneration may affect taxes and benefits

Time cost, i.e. completing reflective practice, explaining jargon, longer meetings and providing help with technical aspects of research

Challenges with scheduling and logistics; limits on engagement due to health problems
Challenges with scheduling and logistics; difficulty identifying and fully involving diverse partners
Patient insights may not be fully representative, as the group was not gender-balanced; risk for team relationships to become obligated, collusive or dependent; risk of personal needs or priorities influencing patient input
Potential impact of challenges, such as managing different perspectives, on the way the project team works together 
Table 2 Reported benefits and risks of involving patients in preparing peer-reviewed publications (Continued)

\begin{tabular}{|c|c|c|c|c|c|c|}
\hline \multirow{2}{*}{$\begin{array}{l}\text { Reference } \\
\#\end{array}$} & \multicolumn{3}{|c|}{ Benefits of patient involvement } & \multicolumn{3}{|c|}{ Risks of patient involvement } \\
\hline & For patients & For others & For the project & For patients & For others & For the project \\
\hline & & & of study findings & & & \\
\hline $\begin{array}{l}{[26]} \\
\text { Blackburn } \\
\text { et al. }\end{array}$ & $\begin{array}{l}\text { Increased knowledge } \\
\text { of own condition, } \\
\text { treatment options } \\
\text { and how to access } \\
\text { services; gain skills, } \\
\text { opportunity for } \\
\text { formal training; } \\
\text { understanding of } \\
\text { research and } \\
\text { research processes; } \\
\text { positive emotional } \\
\text { impact of meeting } \\
\text { new people and } \\
\text { feeling of } \\
\text { contribution; } \\
\text { payment for some } \\
\text { activities }\end{array}$ & $\begin{array}{l}\text { Gain better } \\
\text { understanding of a } \\
\text { condition and } \\
\text { insights on lived } \\
\text { experience; } \\
\text { increased motivation } \\
\text { through the } \\
\text { enthusiasm that } \\
\text { patient contributors } \\
\text { bring; increased } \\
\text { impact of research; } \\
\text { raise profile of } \\
\text { institution and } \\
\text { patient involvement } \\
\text { centre of excellence; } \\
\text { ensure resources are } \\
\text { channeled into } \\
\text { important topics; } \\
\text { guidance on } \\
\text { presenting results to } \\
\text { non-researchers }\end{array}$ & $\begin{array}{l}\text { Improved relevance, } \\
\text { clarity and } \\
\text { accessibility of } \\
\text { materials, surveys } \\
\text { and processes; } \\
\text { setting and } \\
\text { maintaining focus on } \\
\text { the research } \\
\text { question; maintain } \\
\text { realism; increased } \\
\text { recruitment and } \\
\text { follow-up rates; valid- } \\
\text { ation of project and } \\
\text { findings; ensure re- } \\
\text { search is beneficial } \\
\text { to the patient group; } \\
\text { support with data in- } \\
\text { terpretation; promo- } \\
\text { tion of outputs; } \\
\text { generate new or fu- } \\
\text { ture research } \\
\text { questions }\end{array}$ & $\begin{array}{l}\text { Challenging to fully } \\
\text { participate due to } \\
\text { changes in health } \\
\text { status, availability } \\
\text { and other } \\
\text { commitments; } \\
\text { financial costs may } \\
\text { not be reimbursed; } \\
\text { potential impact on } \\
\text { benefits; opportunity } \\
\text { cost for other } \\
\text { activities such as } \\
\text { paid work or } \\
\text { childcare }\end{array}$ & $\begin{array}{l}\text { Time costs, such as } \\
\text { recruitment, } \\
\text { meetings and } \\
\text { communication; } \\
\text { project management } \\
\text { challenges, i.e. if } \\
\text { patient contributors } \\
\text { are unreliable; } \\
\text { opportunity cost for } \\
\text { research time due to } \\
\text { diversion of funds to } \\
\text { patient involvement; } \\
\text { increased pressure } \\
\text { and stress; sensitivity } \\
\text { to criticism }\end{array}$ & $\begin{array}{l}\text { Patient insights may } \\
\text { not be fully } \\
\text { representative as } \\
\text { groups are } \\
\text { homogeneous (i.e. if } \\
\text { patients encourage } \\
\text { friends to } \\
\text { participate); risk of } \\
\text { duplicating efforts, } \\
\text { i.e. patient } \\
\text { involvement and } \\
\text { qualitative work; } \\
\text { patient contributors } \\
\text { may be unreliable; } \\
\text { financial costs such } \\
\text { as travel, meeting } \\
\text { and venue costs, IT } \\
\text { and other } \\
\text { infrastructure, and } \\
\text { payment for patient } \\
\text { contributors to } \\
\text { attend conferences }\end{array}$ \\
\hline $\begin{array}{l}\text { [27] } \\
\text { Barnfield } \\
\text { et al. }\end{array}$ & None reported & $\begin{array}{l}\text { Avoid 'cherry picking' } \\
\text { perception through } \\
\text { patient selection of } \\
\text { relevant PLS for } \\
\text { distribution }\end{array}$ & $\begin{array}{l}\text { Guide selection of } \\
\text { patient-relevant PLS; } \\
\text { improved content } \\
\text { and layout of PLS to } \\
\text { optimize readability } \\
\text { and comprehension; } \\
\text { identify jargon the } \\
\text { research team may } \\
\text { have missed; im- } \\
\text { provements to web- } \\
\text { site for PLS } \\
\text { distribution }\end{array}$ & None reported & $\begin{array}{l}\text { Time and financial } \\
\text { cost related to } \\
\text { conducting focus } \\
\text { groups }\end{array}$ & $\begin{array}{l}\text { Patient insights may } \\
\text { not be fully } \\
\text { representative, as all } \\
\text { patients had a high } \\
\text { level of education } \\
\text { and previous } \\
\text { involvement } \\
\text { experience; personal } \\
\text { experience may } \\
\text { introduce bias, i.e. } \\
\text { preference for using } \\
\text { emotive language }\end{array}$ \\
\hline
\end{tabular}

$P L S$ plain language summary

going to be more involved in sharing results through peer-reviewed publications or regulatory-standard lay summaries, then evidence-based guidance was needed to optimize patient involvement. This was the rationale for our systematic review.

From our systematic review, we found that evidence to guide patient involvement does exist, but it is limited in both quantity and quality. We identified publications that reported the benefits and risks of involving patients in preparing peer-reviewed publications, but we did not identify any publications that reported the effects of involving patients in preparing regulatory-standard lay summaries. Consequently, we have limited our evidencebased recommendations to how to involve patients as authors of peer-reviewed publications. As these appear to be the first set of evidence-based recommendations on this topic, we hope that developers of new or updated guidelines on how to prepare peer-reviewed publications will take our recommendations into account. For example, the Good Publication Practice guidelines (published in 2003 [28], 2009 [29] and 2015 [30]) are one of the most widely used guidelines for publishing industrysponsored research [31], and are due to be updated. As many users of these guidelines will have limited or no experience with involving patients in publications, our evidence-based recommendations may help them maximize the benefits and minimize the risks of embracing this 'innovation' in publications. From our realworld experience of working with patient authors on this systematic review, it would have been helpful for our team (patient and non-patient authors) and our project if we had been able to follow these recommendations, before, during and after preparing this manuscript. We would also encourage guideline developers to address other areas of patient involvement in the publication ecosystem (e.g. inviting patients to serve on publication steering committees, co-creating plain language summaries of publications with patients, thanking patients for their participation in research studies, partnering with patients to raise awareness of research presentations and publications that are most relevant to patients). 
Table 3 Evidence-based best practice recommendations for involving patients as authors

\begin{tabular}{|c|c|}
\hline $\begin{array}{l}\text { Stage of } \\
\text { manuscript } \\
\text { preparation }\end{array}$ & Recommendation \\
\hline \multirow[t]{6}{*}{ Before } & $\begin{array}{l}\text { 1. Ideally, involve patients in the question formulation stage (e.g. involve patients in } \\
\text { publication planning to ensure publications address unmet needs that are relevant and } \\
\text { important to patients) }\end{array}$ \\
\hline & $\begin{array}{l}\text { 2. Identify patient author candidates who are interested in contributing, have relevant } \\
\text { expertise (e.g. lived experience) and can meet authorship criteria (i.e. no guest authorship); } \\
\text { document consented contact details for patient authors in publication management } \\
\text { software }\end{array}$ \\
\hline & $\begin{array}{l}\text { 3. Clarify and document author and contributor roles and responsibilities (e.g. signed } \\
\text { authorship agreements should help ensure expectations are clear and understood; patient } \\
\text { involvement should be substantial; archive signed agreements) }\end{array}$ \\
\hline & $\begin{array}{l}\text { 4. Ensure support for patient authors from non-patient authors, especially the primary au- } \\
\text { thor and publication guarantor }\end{array}$ \\
\hline & $\begin{array}{l}\text { 5. Appoint a designated contact person for patient authors to reach out to with queries } \\
\text { (e.g. a Certified Medical Publication Professional who has publication expertise, project } \\
\text { knowledge and time to support patient authors) }\end{array}$ \\
\hline & 6. Identify relevant publication and patient involvement guidelines that will be followed \\
\hline
\end{tabular}
(e.g. CONSORT, GRIPP2, GPP3)

7. Check that funding facilitates patient author involvement (e.g. upfront payment of travel expenses for author meetings and conference presentations, translator fees if necessary)

8. Prepare a publication timeline that facilitates patient author involvement (e.g. early delivery of materials to review; contingency time for unexpected unavailability - illness, employment, other commitments)

9. Consider providing a publication induction guide and training for patient authors (e.g. plain language summary of GPP3, glossary of publication terms, overview of publication process)

10. Consider how to proactively and systematically evaluate the effect of patient $[19,21,22,24-27]$

$[19,23-25,27]$

$[19,21,23-25,27]$

$[23,25,27]$

$[23,25,27]$

$[19,21,23-27]$

$[21,23-25,27]$

$[19-21,23-25,27]$ involvement (e.g. document feedback via publication management software; administer patient authorship experience tools)

11. Recognize and respect diversity in the authorship team - everyone should contribute and be listened to. Patient authors can provide unique and useful input from their lived experience (e.g. they are not expected to be statisticians, clinicians, medical writers)

12. Be flexible in how patient authors can provide input (e.g. telephone, email, in person)

13. Allow time before, during and after authorship meetings to address concerns and questions about patient authorship - from patient and non-patient authors

14. Provide timely and regular feedback to patient authors on their contributions and group dynamics

15. Consider presenting key results at authorship meetings and in publications that could make it quicker and easier for non-specialists to understand and interpret findings (e.g. use data visualization, flowcharts)

16. Recognize that patient authors may provide stronger contributions if able to provide input in their local language

17. Document, in the manuscript, the involvement and role of patient authors (i.e. identify which authors are patients [e.g. Author Affiliation section] and describe their authorship contributions [e.g. Contributorship section])

19. Involve patients in the publication dissemination plan (e.g. raising awareness of the publication via patient advocacy groups, community and personal networks, social media platforms; contributing to and testing plain language summaries - ensuring cultural and linguistic appropriateness)

20. Encourage continued participation (e.g. patient authors presenting results - target geographically close conferences, leverage remote presentation tools; involvement in follow-up publication projects and publication steering committees)

$[20,21,26,27]$

21. Consider preparing a companion publication on the effect of patient involvement 
Table 4 Report of patient involvement in this systematic review and recommendations project (GRIPP2 Short Form)

\begin{tabular}{ll}
\hline Section and topic & Item \\
\hline 1: Aim & Report the aim of PPI in the study \\
- To collaborate with patients as authors on a systematic review and, based on that review, propose evidence-based rec- \\
ommendations to help other authors (patient and non-patient authors) work together on publications. \\
- To develop a tool, which could be shared with others, to help patient and non-patient authors evaluate their experi- \\
ences of working together on publications. \\
Provide a clear description of the methods used for PPI in the study \\
- Three patient partners were invited to join the research team at the study concept stage. They contributed to the \\
development of the protocol for the systematic review, participated in author meetings, provided feedback on \\
presentations (slide and poster presentations made to European and North American conferences) and manuscript \\
drafts, co-created the plain language summary of the publication and contributed to the development of the Patient \\
Authorship Experience tool. \\
Outcomes-Report the results of PPI in the study, including both positive and negative outcomes \\
- Positive: The patient partners validated that this project would address an important and unmet need, which justified \\
the initiation of the project. Their early and ongoing contributions provided 'real world' insights on the value of patient \\
author involvement (e.g. they raised important points that non-patient authors did not). They provided candid com- \\
ments and constructive criticism, both of which guided and strengthened the project, and they responded to requests \\
for input, even under tight timelines and during holiday periods. Non-patient authors gained personal confidence and \\
professional satisfaction that they were working on a project that would help other teams involve patient authors, ethic- \\
ally and effectively. They also obtained new knowledge and understanding about the complexities of academic \\
publishing. \\
- Negative: Additional time was required to develop new tools (e.g. plain language authorship guidelines, authorship \\
agreements, Patient Authorship Experience tool) to ensure patient authors knew their rights and responsibilities, and to \\
help ensure all team members could share their views and learn from this experience.
\end{tabular}

4: Discussion and conclusions

5: Reflections/critical perspective

Outcomes-Comment on the extent to which PPI influenced the study overall. Describe positive and negative effects - Our patient partners had a critical influence on this project - if they did not see the need for it, then it would not have started. Their passionate belief that patients can and should be authors of publications, not 'just' contributors or readers, inspired the whole team. At the outset of the project, however, we had minimal guidance that was specifically relevant to working with patients as authors. We relied on general 'PPI' guidance documents, which were helpful, but not as specific as we would have liked.

Comment critically on the study, reflecting on the things that went well and those that did not, so others can learn from this experience

- One early issue research teams must address when wanting to involve patients is knowing which patients to invite and then establishing a culture that facilitates early and ongoing contributions. It is clear that if patients are to become authors, not all of them will already possess the skills set needed. For this project, we were in a fortunate position in that we knew patients who were keen to join the project early. Further, these patients were confident and passionate, and they understood that their expertise was valued. These elements contributed to a culture of openness, inclusiveness and efficiency.

- There is recognition among our patient colleagues that if patients wish to become authors, then they will need to meet authorship criteria, that is, play a more substantial role than sense-checking or proofreading (important though these processes are). Inexperience with authorship may create grey areas early on for some patient (co-)authors.

- On reflection, we did make some assumptions (e.g. logistical/technical assumptions about the 'ease' of joining WebEx calls; awareness of publication timelines/processes) that were unwarranted and we have learned how to address these. As we embarked on this project without the benefit of the 21 evidence-based recommendations that we have now proposed, we envisage that future projects will be enhanced by our ability to follow these recommendations.

PPI patient and public involvement, GRIPP2 Guidance for Reporting Involvement of Patients and the Public

From our systematic review, we also found that it was challenging to search the literature and to identify which authors may have been patient authors. Until these two issues can be addressed, research on this topic will not be as efficient or effective as it could be. For example, without the use of standardized keywords to interrogate databases (e.g. MEDLINE), we may have missed eligible publications. The lack of standardized keywords in an emerging area of research is not surprising, and we encourage stakeholders to propose and reach consensus on suitable keywords to enhance future literature searches. We suggest that even the adoption of 'patient author' would help research in this area. We did find that the yield of potentially eligible publications was higher from searching the PCORI's Health Literature Explorer database, as some level of curation had already taken place. We encourage researchers with an interest in this topic to use the PCORI database (https://www.pcori.org/ literature/engagement-literature). In terms of identifying which publications had patient authors, we found that there was no consistent or clear way that patient authors were described. We typically had to review the author affiliation, methods and acknowledgements sections of publications to detect which, if any, authors represented the patient perspective. Even if patient authors were identified, sometimes their listed affiliation was a university or hospital. A subsequent Internet search helped to clarify and confirm their role as a patient author (e.g. leadership role in a patient advocacy group, community advisory board). Understandably, these groups might be 
affiliated with a university or hospital and, quite rightly, this affiliation is given. However, it would help research in this area if such affiliations were complemented by a description of patient representative roles.

In addition to conducting this systematic review and proposing evidence-based recommendations, an important goal of this project was to partner with patients, as authors. We developed a number of tools, with patients, to help facilitate the involvement and evaluation of patient authors in publications and to help share our research findings with the patient community and other interested stakeholders. The PAE tool, with versions for patient authors and for non-patient authors, was a publication-specific adaption of the Patient Engagement Quality Guidance [7]. This guidance was developed by the not-for-profit organization, PFMD, with input from a large and diverse group of stakeholders [7]. We have made the PAE tool available for others to use for free ([18]; Additional Files 4 and 5). We welcome feedback on this tool, and hope that it helps patient and nonpatient authors evaluate how well they worked together and where they might enhance their partnership. We have also developed, with patients and other stakeholders, a Plain Language Summaries of Publications Toolkit (https://www.envisionthepatient.com/plstoolkit/) [32]. We have also made this Toolkit available for others to use for free and welcome feedback on its use. The patient authors of this systematic review played a key role in using this Toolkit to co-create the plain language summary for this systematic review.

Although we have based our recommendations on a systematic review of the literature, we recognize that our research does have limitations and that additions to the literature over time will necessitate updates and expanded perspectives relevant to the expanding evidence. We found nine eligible publications and these all focused on the effects of patient involvement in preparing peer-reviewed publications, not regulatory-standard lay summaries. As more patients become involved in sharing results through publications or lay summaries, we encourage them and their non-patient partners to evaluate their experiences and publish their findings. We need to keep building the evidence base to guide best practice. We also encourage other researchers to expand and update the evidence base (e.g. by examining the grey literature; repeating our search as more papers on this topic are published).

We also acknowledge that while our recommendations are based on evidence, they need to be tested in 'realworld' situations. We have reflected on the recommendations and have recognized how applicable and valuable they would have been to this project, had they been available at the start. We have also started to apply these recommendations in new projects involving patient and non-patient authors, and the initial feedback from patient and non-patient authors has been positive. In terms of global use of these recommendations and the tools we developed, we welcome collaboration with individuals around the world who may be able to assist with translation and cultural adaptations.

\section{Conclusions}

Published evidence on the effects of patient involvement in sharing results from health research studies is limited. From a systematic review of the literature, we have summarized the reported benefits and risks of involving patients in preparing peer-reviewed publications of health research studies. Based on this evidence, we have proposed 21 recommendations for involving patients before, during and after preparing a manuscript for publication. Understanding and learning from the experience of both patient and non-patient authors is equally important, and we piloted the PAE self-assessment tool as an initial step towards unlocking these insights. Researchers, patient partners and publication professionals may draw on these resources as they start or continue to collaborate on ethical, substantial patient involvement in publications.

\section{Supplementary information}

Supplementary information accompanies this paper at https://doi.org/10. 1186/s40900-020-00190-W.

Additional file 1. Infographic plain language summary.

Additional file 2. Summary of search strategies.

Additional file 3. Data extraction spreadsheet for nine publications included in this systematic literature review.

Additional file 4. PAE tool - patient author version.

Additional file 5. PAE tool - non-patient author version.

Abbreviations

PAE: Patient Authorship Experience; PCORI: the Patient-Centered Outcomes Research Institute; PFMD: Patient Focused Medicines Development;

PPI: patient and public involvement

\section{Acknowledgements}

The authors acknowledge the insightful patient representative contributions from Antonio Ciaglia (employed at the International Alliance of Patients' Organizations during study concept development), as well as the professional support from Envision Pharma Group colleagues (literature searching and editorial assistance). The authors also thank the pioneers (patients and their research partners) who have and continue to advocate for ethical and substantial involvement of patients in medicines development - they have inspired our research.

\section{Authors' contributions}

All authors participated in the research, were actively involved in preparing the manuscript and provided approval for submission.

\section{Authors' information}

LA is Patient Partnership Liaison at Envision Pharma Group.

ACW is a Global Business Unit Head at Envision Pharma Group.

BY is a patient author and Professor in the Graduate School of Human

Sciences, Osaka University. 
RS is a patient author and member of the NCRI Consumer Forum and several other UK and European patient groups. He is also Co-Editor-in-Chief of Research Involvement and Engagement.

KS is a patient author and CEO at International Alliance of Patients Organizations.

AS is Value Strategy Lead at Envision Pharma Group.

$\mathrm{RJ}$ is the Medical Director and Founding Partner at Swii.ch Health Ltd.

TG is a Senior Scientific Director at Envision Pharma Group.

KW is the Global Lead for Patient Partnerships at Envision Pharma Group;

Adjunct Professor at the University of Queensland and the University of the Sunshine Coast; and Editorial Board Member for the journal Research Integrity and Peer Review. Author ORCiD iD: 0000-0003-4626-7723.

\section{Funding}

No external grants were used to fund this review. All work was completed on a voluntary basis

All authors acted in a voluntary capacity. Envision Pharma Group provided limited financial support for administrative expenses.

\section{Availability of data and materials}

Original articles are available through their respective publishers, some as open access.

\section{Compliance with ethical standards}

\section{Ethics approval and consent to participate}

Not applicable.

\section{Consent for publication}

Not applicable.

\section{Competing interests}

RS is Co-Editor-in-Chief of Research Involvement and Engagement. KW is an Editorial Board Member for Research Integrity and Peer Review. BY has received consultancy payments from CSL Behring, Shire and Biocryst, and expenses for presentations from CSL Behring, Shire, Biocryst and Takeda. All authors are advocates for greater involvement of patients as partners in medical research, including legitimate authorship of medical research publications.

\section{Author details}

${ }^{1}$ Envision the Patient - Envision Pharma Group, Suite 5.11, 5th Floor, 1 Lyric Square, London W6 0NB, UK. ${ }^{2}$ Alligent EU - Envision Pharma Group, Wilmslow, UK. ${ }^{3}$ Osaka University, Osaka, Japan. ${ }^{4}$ Hereditary Angioedema Japan (Registered NPO), Hyogo, Japan. ${ }^{5}$ Hereditary Angioedema International (Registered NPO/Charity), Fairfax City, VA, USA. ${ }^{6}$ Consumer Forum, National Cancer Research Institute, London, UK. ${ }^{7}$ Research Involvement and Engagement, London, UK. ${ }^{8}$ International Alliance of Patients' Organizations, London, UK. ${ }^{9}$ Swii.ch Health, Manchester, UK. ${ }^{10}$ Curo - Envision Pharma Group, London, UK. ${ }^{11}$ Engage - Envision Pharma Group, Southport, CT, USA.

${ }^{12}$ ProScribe KK - Envision Pharma Group, Tokyo, Japan. ${ }^{13}$ University of Queensland, Brisbane, Queensland, Australia. ${ }^{14}$ University of the Sunshine Coast, Maroochydore DC, Queensland, Australia.

Received: 7 August 2019 Accepted: 31 March 2020

Published online: 24 June 2020

\section{References}

1. Hoos A, Anderson J, Boutin M, Dewulf L, Geissler J, Johnston G, et al. Partnering with patients in the development and lifecycle of medicines: a call for action. Ther Innov Regul Sci. 2015;49:929-39.

2. Bloom D, Beetsch J, Harker M, Hesterlee S, Moreira P, Patrick-Lake B, et al. The rules of engagement: recommendations for successful collaborations between sponsors and patient groups around clinical trials. Ther Innov Regul Sci. 2018;52:206-13.

3. Dewulf L. Patient engagement by pharma - why and how? A framework for compliant patient engagement. Ther Innov Regul Sci. 2015;49:9-16.

4. Yeoman G, Furlong P, Seres M, Binder H, Chung H, Garzya V, Jones RRM. Defining patient centricity with patients for patients and caregivers: $a$ collaborative endeavour. BMJ Innov. 2017;3:76-83.
5. Boutin M, Dewulf L, Hoos A, Geissler J, Todaro V, Schneider RF, et al. Culture and process change as a priority for patient engagement in medicines development. Ther Innov Regul Sci. 2017;51:29-38.

6. International Alliance of Patients' Organizations. Consensus framework for ethical collaboration between patients' organisations, healthcare professionals and the pharmaceutical industry. https://www.iapo.org.uk/ sites/default/files/files/Consensus_Framework-vF.pdf. Published January 2014. Accessed 28 March 2019.

7. Deane K, Delbecque L, Gorbenko O, Hamoir AM, Hoos A, Nafria B, et al. Cocreation of patient engagement quality guidance for medicines development: an international multistakeholder initiative. BMJ Innov. Published Online First: 02 March 2019. https://doi.org/10.1136/bmjinnov-2018-000317.

8. Patients active in research and dialogues for an improved generation of medicines. Paradigm website. https:/imi-paradigm.eu/. Accessed 28 March 2019.

9. Pushparajah DS, Manning E, Michels E, Arnaudeau-Bégard C. Value of developing plain language summaries of scientific and clinical articles: a survey of patients and physicians. Ther Innov Regul Sci. 2018;52:474-81.

10. Woolley KL, Magatova E, Homma Y, Platt EA, Lane P. Who engages with patient-centered, peer-reviewed publications? Tweeting of JAMA patient pages. Poster presentation at the 2017 European meeting of the International Society for Medical Publication Professionals; 17-18 January 2018; London, UK

11. New requirements for patient and public involvement statements in BMJ Open. BMJ Open website. https://blogs.bmj.com/bmjopen/2018/03/23/newrequirements-for-patient-and-public-involvement-statements-in-bmj-open/. Accessed 28 March 2019.

12. Research Involvement and Engagement. BioMed Central Ltd website. https://researchinvolvement.biomedcentral.com/. Accessed 28 Mar 2019

13. European Commission. Regulation (EU) No 536/2014 of the European Parliament and of the Council of 16 April 2014 on clinical trials on medicinal products for human use, and repealing Directive 2001/20/EC. https://ec.europa.eu/health/sites/health/files/files/eudralex/vol-1/reg_2 014_536/reg_2014_536_en.pdf. Accessed 2 May 2019.

14. Moher D, Liberati A, Tetzlaff J, Altman DG, for the PRISMA group. Preferred reporting items for systematic reviews and meta-analyses: the PRISMA statement. BMJ. 2009;339:b2535.

15. Staniszewska S, Brett J, Simera I, Seers K, Mockford C, Goodlad S, et al. GRIPP2 reporting checklists: tools to improve reporting of patient and public involvement in research. BMJ. 2017;358:j3453.

16. Patient Focused Medicines Development. Patient engagement quality guidance. https://wfip.org/wp-content/uploads/2018/05/patientengagement-quality-guidance-1.pdf. Accessed 2 May 2019.

17. National Health Council. Patient-focused drug development recommended language for use in guidance document development. http://www.nationalhealthcouncil.org/sites/default/files/NHC-GA\%20Feb201 7.pdf. Published February 2017. Accessed 2 May 2019.

18. Woolley KL, Arnstein L, Hamoir AM, Lobban D, Stephens R, Yamamoto B. Development and use of 2 tools to facilitate and evaluate patient authorship. Poster presentation at the 15th Annual Meeting of the International Society for Medical Publication Professionals; 15-17 April 2019; National Harbor, MD, USA.

19. Absolom K, Holch P, Woroncow B, Wright EP, Velikova G. Beyond lip service and box ticking: how effective patient engagement is integral to the development and delivery of patient-reported outcomes. Qual Life Res. 2015;24:1077-85.

20. Pollard K, Donskoy AK, Moule P, Donald C, Lima M, Rice C. Developing and evaluating guidelines for patient and public involvement (PPI) in research. Int J Health Care Qual Assur. 2015;28:141-55.

21. Hyde C, Dunn KM, Higginbottom A, Chew-Graham CA. Process and impact of patient involvement in a systematic review of shared decision making in primary care consultations. Health Expect. 2017;20:298-308.

22. Mann C, Chilcott S, Plumb K, Brooks E, Man MS. Reporting and appraising the context, process and impact of PPI on contributors, researchers and the trial during a randomized controlled trial - the 3D study. Res Involv Engagem. 2018;4:15.

23. Pérez Jolles MP, Martinez M, Garcia SH, Stein GL, Mentor Parent Group members, Thomas KC. Involving Latina/o parents in patient-centered outcomes research: contributions to research study design, implementation and outcomes. Health Expect. 2017;20:992-1000.

24. Howe A, Mathie E, Munday D, Cowe M, Goodman C, Keenan J, et al. Learning to work together - lessons from a reflective analysis of a research project on public involvement. Res Involv Engagem. 2017;3:1. 
25. Forsythe L, Heckert A, Margolis MK, Schrandt S, Frank L. Methods and impact of engagement in research, from theory to practice and back again: early findings from the Patient-Centered Outcomes Research Institute. Qual Life Res. 2018;27:17-31.

26. Blackburn S, et al. The extent, quality and impact of patient and public involvement in primary care research: a mixed methods study. Res Involv Engagem. 2018:4:16

27. Barnfield S, Pitts AC, Kalaria R, Allan L, Tullo E. "Is all the stuff about neurons necessary?" The development of lay summaries to disseminate findings from the Newcastle Cognitive Function after Stroke (COGFAST) study. Res Involv Engagem. 2017;3:18.

28. Wager E, Field EA, Grossman L. Good publication practice for pharmaceutical companies. Curr Med Res Opin. 2003;19:149-54.

29. Graf C, Battisti WP, Bridges D, Bruce-Winkler V, Conaty JM, Ellison JM, for the International Society for Medical Publication Professionals, et al. Research methods \& reporting. Good publication practice for communicating company sponsored medical research: the GPP2 guidelines. BMJ. 2009;339: b4330.

30. Battisti WP, Wager E, Baltzer L, Bridges D, Cairns A, Carswell Cl, et al. Good publication practice for communicating company-sponsored medical research: GPP3. Ann Intern Med. 2015;163:461-4.

31. Wager E, Woolley K, Adshead V, Cairns A, Fullam J, Gonzalez J, et al. Awareness and enforcement of guidelines for publishing industrysponsored medical research among publication professionals: the global publication survey. BMJ Open. 2014;4:e004780.

32. Lobban D, Arnstein L, Wadsworth AC, Woolley KL. Plain language summaries of publications: Addressing the HOW via a stakeholder survey and workshop. Poster presentation at the 15th Annual Meeting of the International Society for Medical Publication Professionals; 15-17 April 2019; National Harbor, MD, USA.

\section{Publisher's Note}

Springer Nature remains neutral with regard to jurisdictional claims in published maps and institutional affiliations.

Ready to submit your research? Choose BMC and benefit from:

- fast, convenient online submission

- thorough peer review by experienced researchers in your field

- rapid publication on acceptance

- support for research data, including large and complex data types

- gold Open Access which fosters wider collaboration and increased citations

- maximum visibility for your research: over $100 \mathrm{M}$ website views per year

At $\mathrm{BMC}$, research is always in progress.

Learn more biomedcentral.com/submissions 